\title{
Modelling gravitational waves from precessing black-hole binaries: progress, challenges and prospects
}

\author{
Mark Hannam
}

Received: 12 December 2013 / Accepted: 30 June 2014 / Published online: 14 August 2014 (C) The Author(s) 2014. This article is published with open access at Springerlink.com

\begin{abstract}
The inspiral and merger of two orbiting black holes is among the most promising sources for the first (hopefully imminent) direct detection of gravitational waves (GWs), and measurements of these signals could provide a wealth of information about astrophysics, fundamental physics and cosmology. Detection and measurement require a theoretical description of the GW signals from all possible black-holebinary configurations, which can include complicated precession effects due to the black-hole spins. Modelling the GW signal from generic precessing binaries is therefore one of the most urgent theoretical challenges facing GW astronomy. This article briefly reviews the phenomenology of generic-binary dynamics and waveforms, and recent advances in modelling them.
\end{abstract}

Keywords Numerical relativity $\cdot$ Gravitational waves $\cdot$ Black holes

\section{Introduction}

Our first major physical theory was Newton's law of gravitation, which was powerful enough to describe both the fall of fruit on Earth and the orbits of planets in the solar system. Newton's calculations of two-body dynamics from one general law were the central triumph of the scientific revolution. Now Newton's theory of gravitation has been upgraded to Einstein's general theory of relativity, where two-body dynamics can become far more complex, and may once again be the catalyst for discoveries about the universe. The motion of two orbiting bodies has been modified in two important ways. (1) All acceleration generates gravitational waves; those generated by the orbital motion carry away energy, which causes the orbital separation to slowly decrease, and

M. Hannam ( $\square)$

School of Physics and Astronomy, Cardiff University, The Parade, Cardiff CF24 3AA, UK

e-mail: mark.hannam@astro.cf.ac.uk 
the bodies to spiral together. (2) The angular momenta of the bodies are no longer individually conserved, and in general their individual angular momenta (spins), and the orbital plane of the binary, all precess. These effects are weak when the two bodies are far apart (being only a small correction to the Newtonian description), but for compact objects like neutron stars and black holes, which may continue to orbit up to very small separations, the precession effects can lead to wild complex dynamics, before the inspiral terminates in a collision that produces an intense final burst of gravitational radiation.

The extreme physics of black-hole and neutron-star inspiral and merger may soon be measurable by the new gravitational-wave observatories Advanced LIGO (aLIGO) and Advanced Virgo (AdV), which are due to come online in 2015-2016 [1,2]. The primary goal of these experiments is to make the first direct detection of gravitational waves, and our current understanding of astrophysics suggests that "compact binary coalescences" are most likely to provide the first detections; indeed, when aLIGO and AdV reach their design sensitivity around 2018 [3], they may observe neutron-star and black-hole mergers on a daily basis-although the uncertainty in astrophysical event rates is so large that they are also consistent with no detections at all [4].

Our ability to observe the gravitational waves from binary mergers, and in particular to measure the binary's properties from the GW signals (their masses and angular momenta, and the binary's location in the sky and distance from Earth), all depend on theoretical predictions of the signals produced by all possible binary configurations.

The GW signal from the slow inspiral can be predicted by a post-Newtonian (PN) expansion of the Einstein equations [5]. The late inspiral and merger requires numerical solutions of the full nonlinear Einstein equations [6]. Both approaches are necessary because the PN approximation breaks down as the black holes approach merger, and numerical-relativity (NR) simulations are too computationally expensive to generate waveforms that cover the thousands of orbits that could be observable by aLIGO and AdV. The construction of complete inspiral-merger-ringdown (IMR) models for generic-binary configurations in principle require NR simulations across a sevendimensional parameter space (explained in more detail in Sect. 2). By 2010 models had been produced for a subset of binary configurations that do not precess (the blackhole spins are parallel to the binary's orbital angular momentum, as I will describe later). In these configurations the waveforms have a simple structure, and most of the signal power resides in the dominant waveform harmonic, and we need only model its (relatively simple) amplitude and phase. These models required 20-30 numerical simulations [7-11]. A simple counting argument suggests that extending these models to generic configurations would require tens of thousands of simulations. In addition, the precession spreads the signal power across other waveform harmonics, making the modeling of these waveforms (not just describing a single waveform, but also the subtle waveform variations with respect to binary configurations) far more challenging. In the strong-field regime near merger the detailed phenomenology of generic binaries can be determined only after the numerical simulations have been performed, so a judicious sampling of that parameter space is difficult to estimate a priori. For these reasons, modeling the GW signal from generic binary mergers has become the most urgent theoretical challenge facing gravitational-wave astronomy. 
The statements above summarize our understanding of the problem around 2010. Since then a number of important studies have shown how to factorize out the complex precession effects from the waveforms, and have indicated that we can, to a good approximation, produce generic models by applying a time-dependent rotation to non-precessing-binary waveforms, based on the precessional dynamics of the particular configuration we wish to describe. In other words, we can "twist up" the non-precessing-binary models that we already have to produce generic models. This is an approximation that does not remove all of the issues in generic-binary waveform modeling, and so far includes only a rudimentary treatment of the merger and ringdown phase-but these results nonetheless mark a huge step forward in the modeling of generic systems, and suggest that a solution sufficient for the needs of gravitationalwave astronomy may be possible in the near future.

The purpose of this review is to expand on the above. The focus is on the goal of constructing IMR models for generic-binary systems. I will summarize the basic phenomenology of generic-binary dynamics and waveforms (Sect. 2), the PN description of the inspiral (Sect. 3), and then IMR models from the current non-precessing-binary models (Sect. 4) through to the latest results in generic-binary modelling (Sect. 5). At the end I will discuss some of the remaining issues and challenges (Sect. 6).

\section{The effect of spin on binary dynamics and waveforms}

The effect of spin on the dynamics of two compact bodies was first considered by Barker and O'Connell [12]. Extensive reviews of early work are given in Refs. [13,14]. The phenomenology of the binary dynamics and the gravitational-wave signals are discussed and illustrated in detail by Apostolatos et al. [15] and Kidder [16]. In this section I summarize these effects in the order of their impact on the GW signal, which mimics the progress of GW-modelling efforts in recent years.

In the following we consider two black holes of masses $m_{1}$ and $m_{2}$, and refer to the total mass as $M=m_{1}+m_{2}$. The mass-ratio between the two black holes is $q=m_{1} / m_{2}$, where $m_{1}>m_{2}$ and $q>1$, although other conventions are also used in the literature. A less ambiguous indicator of the mass ratio is $\eta=m_{1} m_{2} / M^{2}$, which is independent of whether we define $q>1$ or $q<1$. If the black-hole spinangular-momenta are $\mathbf{S}_{1}$ and $\mathbf{S}_{2}$, then the dimensionless spins are $\chi_{1}=\left|\mathbf{S}_{1}\right| / m_{1}^{2}$ and $\chi_{2}=\left|\mathbf{S}_{2}\right| / m_{2}^{2}$, and $\chi_{i} \in[0,1]$, where $\chi=1$ corresponds to an extreme Kerr black hole.

The mass ratio and spin vectors give us a seven-dimensional parameter space. In this work we consider only black-hole binaries following non-eccentric inspiral, and so eccentricity does not add to our total parameter count. We do this because we expect that by the time most binaries enter the aLIGO and AdV sensitivity bands, any eccentricity present at the binary's formation will have essentially radiated away [17]. We also do not need to include the total mass of the binary in analytic or numericalrelativity calculations, because this defines the overall length scale of the spacetime, and can be factored out of any solution. In applications of our waveforms to GW astronomy, however, the mass scale must be included, as well as the particular form of 

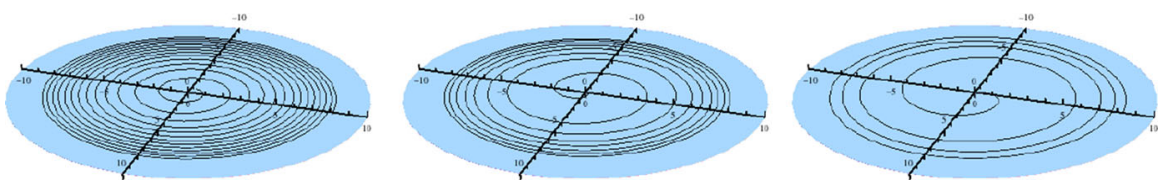

Fig. 1 Phenomenology of inspiral for aligned-spin systems. The figures show the motion of one black hole in a mass-ratio 1:4 binary, from a separation of $D \approx 8 M$ through to merger, where $M$ is the total mass of the binary. For a binary with total mass 25 times that of our sun, $8 M$ would be approximately $300 \mathrm{~km}$, and the inspirals shown would all take less than a quarter of a second. The central figure depicts a binary made up of nonspinning black holes; in the left figure the spins are parallel to the orbital angular momentum, and in the right figure they are in the opposite direction; the spins are $\chi_{i}=0.75$. The "hang-up" effect aligned with spins is clear

the GW signal for a given binary orientation, polarization, distance and sky positionbut these intrinsic parameters do not need to be included in the waveform model.

\subsection{Non-precessing systems}

If two black holes do not spin, or if their spins are parallel to the orbital angular momentum of the binary, then the direction of the orbital plane of the binary is fixed. The black holes follow non-eccentric, but slowly decaying orbits. The rate of decay is determined by the loss of energy due to gravitational radiation, and this is a function both of the black-hole masses, but also their spins. In a post-Newtonian expansion of the equations of motion, we can see that if the spins are parallel to the orbital angular momentum, then the rate of energy loss is reduced, and the black holes inspiral more slowly, e.g., see Eqn. (1) in Ref. [18]. If the spins are in the opposite direction to the orbital angular momentum, then the black holes inspiral more quickly. This effect is illustrated in Fig. 1 for three spin configurations of a mass-ratio 1:4 binary. As the inspiral proceeds, angular momentum is radiated from the system and the orbital angular momentum of the binary decreases; although the spin magnitudes can vary in some PN treatments (see, for example, Ref. [19]), this effect is far smaller than other uncertainties in the PN calculations, and in all waveform models discussed here the spin magnitudes are treated as constant.

\subsection{Precession}

When the spins are not aligned with the orbital angular momentum, then spin-orbit and spin-spin couplings lead to precession of the spins and orbital plane. This is a purely relativistic effect: in Newtonian physics the individual angular momenta of the two bodies, and of the total system, are all individually conserved. In the absence of gravitational radiation, the direction of the total angular momentum $\hat{\mathbf{L}}$ would remain fixed, and the orbital and spin angular momenta all precess around it, i.e., $\mathbf{J}=0$ and so $\dot{\mathbf{L}}=-\dot{\mathbf{S}}$. For single-spin systems it is possible to estimate the rate of precession, i.e., the angular speed of $\hat{\mathbf{L}}$ about $\hat{\mathbf{J}}$, to be $\Omega_{p} \propto J / r^{3}$, where $r$ is the separation of the binary [15]. When we include radiation-reaction effects, we find that in most cases the direction of $\hat{\mathbf{J}}$ remains approximately fixed; it is certainly the closest we have to a 

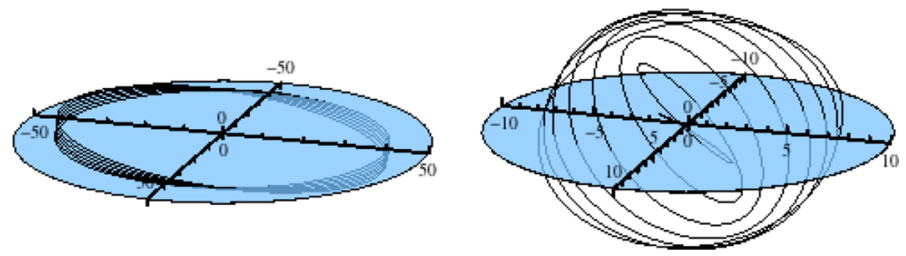

Fig. 2 As in Fig. 1, but for a mass-ratio 1:3 binary, where the larger black hole's spin is $\chi_{1}=0.75$, and is perpendicular to the orbital angular momentum, leading to significant precession. The precession over several orbits is mild at large separations (left), but leads to complicated dynamics near merger (right)

fixed direction during the inspiral. Figure 2 shows an example of the orbital motion of one of the black holes in a precessing binary.

The exceptions to this description are configurations where the black-hole spins are almost anti-aligned with the total angular momentum, such that $|\mathbf{J}|=|\mathbf{L}+\mathbf{S}| \approx 0$. Now there is no fixed precession axis, and the orbital plane "tumbles" in space [15]. Loss of angular momentum through gravitational-wave emission means that this situation cannot persist indefinitely; $|L|$ decreases while $\left|S_{1}\right|$ and $\left|S_{2}\right|$ remain fixed, and $|J|$ does not remain small. For this reason the phenomena is known as transitional precession. Although a fascinating effect, transitional precession will be rare in binaries that are observable by GW detectors: it requires very particular spin configurations, which would have to be met while the binary is within the detectors' sensitivity band.

\subsection{Waveforms}

We can see the effect of precession on the gravitational-wave signal simply by considering the leading-order quadrupole contribution, which takes into account only variations in the moment of inertia of the binary, i.e., the accelerations of the two bodies. For an orbiting binary, the wave signal estimated from the quadrupole approximation is directed predominantly perpendicular to the orbital plane. This is why, in a non-precessing binary, the GW signal is strongest directly above or below the plane of the binary. This dominant contribution to the signal can be represented entirely by the $(l=2,|m|=2)$ spin-weight $(-2)$ spherical harmonics, which means that the signal will be the same for all orientations of the binary, up to an overall amplitude factor and phase offset. This simplifies both the modeling of these signals - to a good approximation we can focus on only the $(2, \pm 2)$ modes - and searches for them in detector data. Most searches comb through the data with a template bank of theoretical waveforms, and in these non-precessing configurations the search template bank does not need to include waveforms that vary with respect to binary orientation and detector polarization, because these do not change the functional form of the waveform; the search only need cover a parameter space of the two masses and spin magnitudes. (To date actual searches in detector data have considered only nonspinning black holes, so the template banks have been two-dimensional; the most recent examples are Refs. [20,21]).

When the orbital plane precesses, the principal emission direction will also precess. Now the waveform structure is more complicated. If the inclination angle between $\mathbf{L}$ 


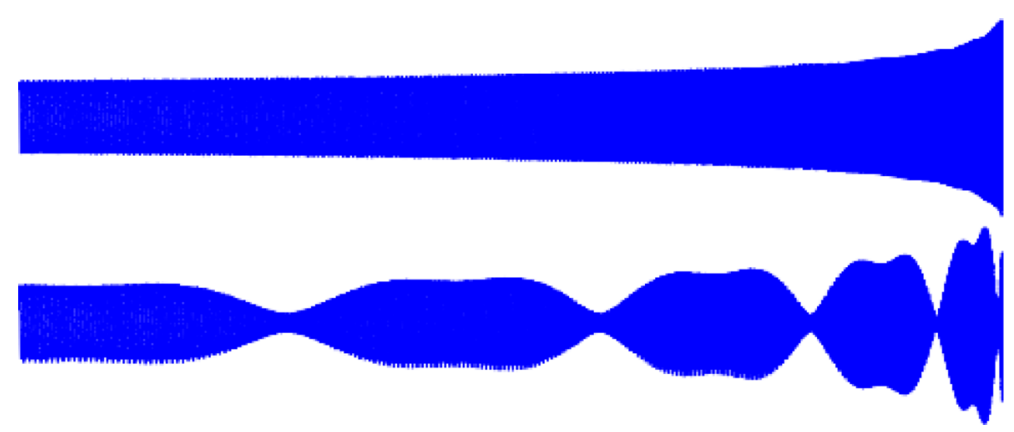

Fig. 3 Gravitational waveforms for the mass-ratio 1:3 system shown in Fig. 2. In the upper figure the observer is oriented approximately along the direction $\hat{\mathbf{J}}$, and the effect of precession on the waveform is minimal. In the lower figure the observer is perpendicular to $\hat{\mathbf{J}}$, and significant modulation effects are visible

and $\mathbf{J}$ is small, and the wave signal is decomposed with respect to spin-weighted spherical harmonics ${ }^{-2} Y_{\ell m}(\theta, \phi)$ that are defined such that the $\theta=0$ direction (the $\hat{z}$ axis) is aligned with $\mathbf{J}$, then the signal can again be represented to a good approximation by only the $(2, \pm 2)$ modes. But there will now be a quadrupole contribution to other $l=2$ harmonics, which grows as the $\hat{\mathbf{L}}-\hat{\mathbf{J}}$ inclination angle is increased. Furthermore, the signal will now vary significantly depending on the relative orientation of the binary to the detector; now that the dominant quadrupole contribution to the signal is made up of all $l=2$ harmonics, the variations in the waveform with binary orientation cannot be treated as an overall amplitude factor in a search (although of course the orientation-dependence of the waveform can be described analytically, by varying $(\theta, \phi)$ in the spherical harmonics).

This is illustrated in Fig. 3, which shows the GW signal from a precessing massratio $1: 3$ binary from two different orientations, where the observer is aligned with $\hat{\mathbf{J}}$, and where the observer is perpendicular to $\hat{\mathbf{J}}$. We might loosely refer to these orientations as "face-on" and "edge-on", but this is misleading. Since $\hat{\mathbf{L}}$ precesses around $\hat{\mathbf{J}}$, the average direction of the normal to the orbital plane lies approximately along $\hat{\mathbf{J}}$, but the observer is in fact never exactly face-on to the binary. Similarly, in configurations with a large $\hat{\mathbf{L}}-\hat{\mathbf{J}}$ inclination angle, we may choose orientations where the binary alternates between being face-on and edge-on as the precession progresses. Nonetheless, the waveform viewed from the $\hat{\mathbf{J}}$-aligned orientation appears very similar to one from a non-precessing binary, and indeed would be difficult to distinguish in a GW observation. It is also important to note that it is the waveform phase, not amplitude, that has the dominant effect in waveform measurements, and these figures are a meaningful illustration of the differences in the waveforms with respect to orientation only because modulations in waveform amplitude and in phase are closely related. If that were not the case and these two waveforms had the same phase evolution, these two waveforms would be difficult to distinguish if used in a search template bank.

Having summarized the phenomenology of binary dynamics and waveforms, I will move on to the details of waveform modelling. 


\section{In the beginning: inspiral}

The early inspiral of the binary, from which thousands of GW cycles may be visible to ground-based detectors, can be described by a post-Newtonian (PN) approximation to the Einstein equations. A detailed summary of PN calculations of the orbital dynamics and waveforms is beyond the scope of this short review; the reader is referred to Blanchet [5] for a thorough review of the current status of PN calculations, and to Apostolatos et al. [15] and Kidder [16] for detailed illustrations of the phenomenology of precessing systems.

The key ingredients in the construction of most PN waveforms for generic systems following adiabatic quasicircular inspiral are: (1) The orbital frequency evolution, which depends on the total mass $M$, the mass ratio $\eta$, and the components of the bodies' spins $\mathbf{S}_{i}$. (2) The precessional dynamics, expressed as equations of motion for $\mathbf{L}$ and $\mathbf{S}_{i}$. (3) The waveform polarizations as a function of the orbital motion.

Ingredients (1) and (2) arise from the equations of motion for the conservative dynamics (i.e., without gravitational radiation), plus the gravitational-wave flux terms. In the equations of motion the non-spinning terms are known up to 3.5PN order [5], and the energy for circular orbits up to 4PN [22]. Spin-orbit coupling terms are also known up to 3.5PN order [23,24]. Spin-spin couplings are known to next-to-leadingorder (NLO, 3PN), and some terms are also known to next-to-next-to-leading-order (NNLO, 4PN) [25, 26]. In the flux NNLO (3.5PN) spin-orbit terms are known [27], and "tail" effects to next-to leading order (4PN) [28], while spin-spin effects are known only to leading order (2PN) [29].

Note that the expressions for the GW phasing at a given PN order are not unique, and that depending on the way the PN terms are truncated in the calculation of a given "approximant", the results will differ in the error terms at higher PN orders.

The GW polarization amplitudes are known to 3PN order in nonspinning terms [5], although the 3.5PN contribution to the $(\ell=2, m=2)$ harmonic is also known [30]. The spin terms are known only to their respective (spin-orbit, spin-spin, and tail) leading orders, which means that the highest PN order for which all spin terms are consistently known is $2 \mathrm{PN}[31]$.

Note that some of these terms were calculated only in the last year. Some higherorder spin terms are likely to appear in the near future, while others (for example calculations to $4 \mathrm{PN}$ order in the flux) will be far more challenging. The convergence properties of the PN expansion are poorly understood, and it is not clear how many more terms are necessary to reach the desired accuracy for GW observations. Our current understanding, based on comparisons of all of the available approximants, is that nonspinning PN waveforms are sufficiently accurate for low-mass systems where the merger and ringdown are at the high-frequency edge of the detectors' sensitivity band (i.e., for detection of neutron-star binaries [32]), but that the uncertainty in spinning PN waveforms from low-mass systems (in particular neutron-star-black-hole binaries) is large enough to cause some reduction in detection efficiency [33,34]. The accuracy of PN waveforms for many non-precessing-binary configurations has been quantified near merger (where the PN waveforms are least accurate) by comparison with fully general-relativistic NR calculations [35-39], and these comparisons have provided 
independent validation for the use of PN waveforms during the inspiral, up to a few orbits before merger.

The accuracy of the PN waveforms could in principle be improved by an appropriate resummation of the series. This has been achieved to a remarkable degree by the effective-one-body (EOB) programme. The conservative dynamics are mapped to the motion of a test particle in an effective metric, which is the Schwarzschild metric deformed by the symmetric mass ratio $\eta$. This was first proposed by Buonanno and Damour [40]. In follow-up work the flux terms responsible for radiation reaction were also resummed (initially by Padé resummation) to produce the full inspiral dynamics, and the GW signal was constructed at leading (quadrupole, or "restricted") PN order [41]. The inspiral waveform was then matched to a ringdown waveform at the "light ring" (an unstable orbit for massless particles), motivated by test-mass and close-limit-approximation results. Those results constituted the first prediction of a full inspiral-merger-ringdown (IMR) waveform.

The first EOB model for non-spinning binaries in 2000 was followed in 2001 by an extension to spinning objects [42], using an effective Kerr metric, although it was later found that similar accuracy can be obtained by simply augmenting the non-spinning EOB Hamiltonian with standard PN spin terms [43].

These models were based on heuristic arguments for the phenomenology of the merger (in particular that the inspiral makes a rapid transition to ringdown at a certain point, and this point can be estimated as the light ring), but had to wait for full numerical simulations in 2005 [44-46] for confirmation and further extension.

\section{Start simple: complete models for non-precessing binaries}

The first IMR model calibrated to numerical simulations was produced using a different ("phenomenological") approach [47], motivated by the need in GW searches for computationally efficient frequency-domain models. This work proposed separate phenomenological ansatzes for the GW phase and amplitude, based on PN results for the inspiral, empirical observations of the late-inspiral/merger phase [48], and ringdown results from perturbation theory. The coefficients in these ansatzes were determined from NR simulations of nonspinning binaries, and these in turn were used to produce an analytic fit of the coefficients across the parameter space. One of the key results of this work (besides the construction of the first NR-based IMR model), was that the phenomenological coefficients varied almost linearly with respect to the binary's mass ratio, suggesting that a complete nonspinning-binary model could be constructed from only a small number $(\sim 5)$ of NR simulations.

Spinning binaries present a far greater challenge, with six additional parameters (the components of the two spin vectors). Modelling efforts began first with the simple subset of non-precessing binaries, where the spins are parallel or anti-parallel to the binary's orbital angular momentum. As described earlier, the dominant effect of the spin in these cases is to increase or decrease the rate of inspiral; it will also modify the spin of the final black hole, since to first approximation $J_{\text {final }}=L+S_{1}+S_{2}$, where we consider the orbital and spin angular momenta just prior to merger [49]; more accurate expressions of the final spin based on NR simulations have been calculated 
for both non-precessing [50] and generic binaries [51]. Besides these spin effects, the basic structure of the waveforms remains unchanged from nonspinning configurations, which greatly simplifies the modelling.

In PN theory we also find that the dominant spin effect on the inspiral rate arises from a combination (essentially a weighted sum) of the two spins [52]. Ajith et al. realized that this allows us to construct a simple non-precessing-binary model with only three physical parameters $\left(M, \eta, \chi_{\text {eff }}\right)[7,9]$, where $\chi_{\text {eff }}$ is a total-effective-spin, $\chi_{\text {eff }}=\left(m_{1} \chi_{1}+m_{2} \chi_{2}\right) / M$, which is closely related to the "reduced spin" combination that appears in PN expressions [52,53]. Such reductions in the parameter space are important: they identify the dominant physical effects in the waveforms, and therefore the physical parameters (or, more often, and unfortunately, combinations of physical parameters) that could be measured in GW observations; they reduce the number of templates necessary in a GW search, which is crucial to make such searches computationally feasible; and they potentially reduce the parameter space of necessary NR simulations to construct models. The total-effective-spin was used in the first NRbased non-precessing-binary IMR model, which was proposed in Ref. [7] (using 26 NR simulations) with only minor modifications to the procedure introduced in Ref. [54], and later refined to use the most accurate PN expressions for the inspiral [9].

In the case of the EOB IMR models, it was found that the EOB dynamics, while they display much-improved accuracy over the original PN calculations, do not agree sufficiently at the known PN order with fully general-relativistic NR results near merger. (Note that while the phenomenological models require the PN waveforms to be sufficiently accurate up to only $\sim 10$ orbits before merger, when they make a transition to an NR-calibrated model, EOB models require the EOB dynamics to be accurate all the way to merger. One's definition of "sufficiently accurate" depends on the application, and remains a subject of study; see Sect. 6 for more). To overcome this, a "pseudo-4PN" term was introduced and calibrated to equal-mass nonspinning NR simulations [55]. Further adjustable parameters were later introduced into the EOB Hamiltonian, the flux terms, the resummation of the waveform modes, and (when extending to spinning systems) the matching time to ringdown modes; all of these parameters were fit to NR simulations.

Nonspinning "EOBNR" models were introduced shortly after the first phenomenological model, and successively refined with additional NR simulations, further adjustable parameters, and a more accurate resummation (factorization) of the waveform modes [56,57]; the most recent nonspinning-binary model is presented in Ref. [58], and a model with harmonics beyond $\ell=2$ is presented in Ref. [59]. A first non-precessing-binary model was introduced in 2010 [8]. Unlike the phenomenological models, it included the magnitudes of both black-hole spins. It was updated in 2012 [10] based on an improved spinning EOB Hamiltonian [60], and more recently calibrated to a larger set of 27 numerical simulations [11].

The EOBNR models are currently computationally expensive to generate, which limits their practicality for GW parameter estimation. However, there have been recent efforts to use reduced-order modelling techniques to construct efficient and accurate surrogate models. Test models of the nonspinning EOBNR model have been constructed over a series of mass-ratio intervals [61], as well as a complete reduced-order model of the non-precessing SEOBNR (version 1) [62]. Related methods have also 
been applied to phenomenological models, for example in Ref. [63] singular-valuedecomposition (SVD) methods were used to calibrate a reduced basis of nonspinning phenomenological waveforms against a set of NR waveforms [63]. These methods are likely to play a key role in providing usable waveform models for $\mathrm{GW}$ astronomy applications in the coming years.

\section{The final challenge: generic binaries}

The leap from non-precessing to generic binaries is large: the phenomenology of the binary dynamics and waveforms becomes far more complex; in addition, the parameter space grows from three (or two) intrinsic parameters, to seven. Let us enumerate the parameters more clearly. A number of parameters of the GW signal can be modified (or added) analytically to a single waveform, and so do not need to be included in an underlying model, or in numerical simulations to construct the model. These include the total mass of the system, which is an overall scale factor in the waveforms; the orientation of the binary with respect to the detector, or the orientation of a detector with respect to the source on the sky; and the distance of the source from the detector. We may also trivially apply a time shift to a waveform, and an overall physical rotation to the entire binary. (For nonprecessing binaries, this rotation is equivalent to a constant phase offset in the waveform). We are now left to model the waveforms with respect to the binary's mass ratio, and the three components of each black hole's spin. Note that, although there may be approximate degeneracies between these parameters, the essential parameter space remains formally seven-dimensional in non-eccentric binaries.

Several approximate degeneracies between configurations may simplify the modelling problem, but it is important to emphasize that they are only approximate, and in particular may break down completely through merger and ringdown. We've already seen one example, the total effective spin $\chi_{\text {eff }}$ in the phenomenological non-precessingbinary models. In the ringdown it is instead the final total spin that characterizes the binary, and so the validity of the effective total spin in parametrizing the waveforms weakens in higher-mass binaries, where the detector is more sensitive to the merger and ringdown $[64,65]$.

Another example is an approximate degeneracy between cases where the components of the black-hole spins in the orbital plane have the same relative orientation, i.e., the same angle between the in-plane spin components: if we rotate both spins in the orbital plane, to leading order this does not change the waveform beyond an overall phase change that can be incorporated analytically. This near-equivalence has been exploited in the past in PN models (see, for example, Ref. [18]), but it is not an exact degeneracy because a rotation of the spins and a phase change do not commute. An easy way to see this is to consider the "superkick" configuration [66-68]. The black holes have equal spins, both in the orbital plane, and oppositely directed, such that the orbital plane does not precess, but does bob up and down due to the emission of linear momentum out of the plane. When this effect terminates at merger, the final black hole recoils out of the orbital plane. The magnitude and direction (up or down) of the recoil will vary with the initial direction of the spin vectors in the plane. The final 
recoil is an unambiguous physical effect (it is independent of the binary and observer orientations) that depends on the initial spin direction, and cannot be removed by a mere phase change. During inspiral the effect of this spin angle on the waveform is minimal, and can be ignored to a good approximation, but not during merger and ringdown.

In order to develop a general procedure to model generic systems through merger and ringdown, we are faced with two problems. The first is to choose the configurations to simulate numerically. If we make the naive estimate that, based on the non-precessing-binary phenomenological models, we need $\sim 4$ simulations in each direction of the parameter space, then for our seven-dimensional parameter space, we will need on the order of tens of thousands of simulations. We do not expect this to be computationally feasible in the next few years. The second problem is that, given these waveforms, we need a means to model the far more complex waveform structure of precessing systems. Prior to 2010, it was not clear how to solve either of these problems. A preliminary effort was made to bridge the gap between generic inspiral PN waveforms and the ringdown [69], but that model's physical fidelity was not tested beyond a small number of equal-mass binaries, and it has not lead to a general approach to generic-binary modelling.

\subsection{Quadrupole alignment}

The key to solving the second problem - the complicated mode structure and amplitude and phase modulations of precessing-binary waveforms-lies in the qualitative description of waveforms in Sect. 2.3. The dominant GW emission directions are perpendicular to the plane of the binary, and in a coordinate system aligned with that direction, most of the signal's power resides in the $(\ell=2,|m|=2)$ spin-weighted spherical harmonics. Although a full description of the GW signal is more complicated, we nonetheless expect that, so long as our coordinate system is appropriately aligned with respect to the orbital plane, then the GW signal will be relatively simple. In other words, if we describe the waveform in a "co-precessing" coordinate system, then generic-binary waveforms will take on a far simpler form than in a real observer's inertial frame.

A co-precessing frame was first used by Buonanno et al. [18] in the study of postNewtonian inspiral waveforms. They observed that the waveforms were indeed simpler in this frame; in fact, the waveform amplitude and phase modulations were removed. This observation lead them to extend the representation proposed in Ref. [70] of a generic waveform as a simple "carrier waveform" modulated by two time-dependent polarization tensors. Ultimately, though, the co-precessing frame was used primarily as a technical tool in the generation of PN waveforms, in particular later in Ref. [71], and to motivate a model that includes a carrier wave plus modulations [18].

Eight years later, a co-precessing frame arose again as a consequence of the work of Schmidt et al. [72]. Their original goal was to track the precession of a binary from the gravitational-wave signal alone, since time-delay effects make it difficult in numerical simulations to match the orbital precession at the binary source to the corresponding effects in the waveform at the observer. They noted that, since the dominant GW 
power lies in the quadrupole $(\ell=2,|m|=2)$ modes when the coordinate system is appropriately aligned, it follows that the instantaneous direction of the orbital angular momentum could be identified with the orientation of the coordinate system for which the $(\ell=2,|m|=2)$ modes of the waveform were maximized. In doing so, the precession can be tracked from the GW signal alone. It turns out that this direction is not exactly normal to the orbital plane, but in fact follows the direction of the orbital angular momentum; in general the two do not coincide, as can be seen by a simple analysis of a PN expansion of the orbital angular momentum. They referred to this accelerating frame of reference as the "quadrupole aligned" (QA) frame.

An alternative co-precessing frame was proposed in 2011 [73], defined by the principal axes of the GW signal. It was later shown in Ref. [74] that this reduces to the QA frame if the principal-axis calculation is restricted to only the $\ell=2, m= \pm 2$ harmonics in the co-precessing frame. The main purpose of Ref. [74] was to complete the construction of a unique co-precessing frame with a third rotation. This corresponds to a time-dependent phase shift in the co-precessing waveform, and its importance is clear in examples of long (PN) waveforms, where the additional phase shift is necessary to recover a smooth monotonic frequency evolution in the co-precessing waveform.

\subsection{Mapping to non-precessing binaries}

The most important consequence of the QA frame was the observation that the waveforms in the QA frame not only have the same simple (non-modulated) form as nonprecessing-binary waveforms, but that the entire mode structure of the corresponding non-precessing-binary waveform seems to have been reproduced.

The identification between quadrupole-aligned (QA) and non-precessing-binary waveforms was observed in Ref. [72] for the mode amplitudes. As we have discussed previously, it is not the waveform amplitude, but the phase, which is most important in GW observations and measurements. But, remarkably, Schmidt et al. later found that a correspondence between each QA waveform and the $(2,2)$ mode of a non-precessingbinary counterpart could also be extended to the phase, and therefore to the entire waveform [75]. They saw that, to a good approximation, the inspiral part of a QA waveform is the same as the waveform produced by a non-precessing system with the same values of the non-precessing spin components; good phase agreement with other modes was also found for the inspiral in Ref. [65]. In fact, since the motivation was to extend the single-effective-spin phenomenological models to precessing systems, the authors tested their claim against systems with only the same value of the total effective spin $\chi_{\text {eff }}$ defined from the aligned-spin components, and so the identification was between systems with the same values of $\left(M, \eta, \chi_{\mathrm{eff}}\right)$.

They also noted a crucial corollary. Let us split the spins into those components parallel to the orbital angular momentum, $\chi_{\|}$, and those perpendicular to the orbital angular momentum, i.e., approximately in the plane of the binary, $\chi_{\perp}$. If we can factorize a precessing-binary waveform $h\left(M, \eta, \chi_{\|}, \chi_{\perp}\right)$ into a non-precessing-binary waveform $h\left(M, \eta, \chi_{\|}\right)$(where the common parameters are the same in both waveforms), plus a rotation defined by the precession dynamics, then the modelling problem has been reduced to finding a model for the precession angles alone. Given such 
a model, we can use it to construct any generic-binary waveform out of its underlying non-precessing-binary waveform. The efficacy of this approach is demonstrated for one example in Ref. [75], and this prescription is effectively applied in Ref. [76] to produce a closed-form frequency-domain generic-binary inspiral model.

This identification is not expected to hold through ringdown, because the ringdown waveform is determined by the final black hole's spin, and this depends more strongly on the in-plane components of the individual black-hole spins prior to merger. One configuration was studied in Ref. [75], and it was found that the identification seems to hold up until the beginning of the ringdown. Pekowsky et al. [65] examined merger waveforms in more detail. They showed that the QA merger-ringdown waveform once again agrees well with a non-precessing counterpart, but, consistent with Ref. [75], there is no simple identification between the QA waveform and its non-precessing counterpart, as there is in the inspiral regime. They also performed a test complementary to that in Ref. [75]: for a set of numerical configurations they applied the appropriate timedependent rotation to a phenomenological non-precessing model, and compared the new "synthetic" waveform to the numerical-relativity merger-ringdown waveform, and again found good agreement. They argued, however, that since this procedure neglects some of the physics of the generic systems (for example, the breaking of the symmetry between the $(2,2)$ and $(2,-2)$ modes of the co-precessing waveform), IMR models based on twisting up non-precessing waveforms may not be sufficiently accurate for GW parameter estimation.

\subsection{Twisting waveforms}

Following these preliminary studies, generic-binary models now have been produced by twisting up non-precessing waveforms.

The "PhenomP" model [77] provides a simple implementation of the procedure proposed in Ref. [75]. This model uses one of the phenomenological models as the underlying non-precessing-binary model. The model of the precessional dynamics is provided by PN results; these are closed-form frequency-domain expressions for the inclination angle between the orbital and total angular momenta, and the precession angle, augmented by recent next-to-next-to-leading order (NNLO) calculations [27]. The ringdown non-precessing-binary model is modified based on predictions of the final black hole's spin [51]. Finally, the authors claim that they can adequately model generic precessing waveforms using only three physical parameters. The symmetric mass ratio and effective total spin (parallel to the orbital angular momentum) carry over from the non-precessing-binary model, and follow from the results in Ref. [75]. For the remaining four in-plane spin components, they exploit the observation that the dominant precession effects can be parameterized by only one "precession spin" parameter, $\chi_{p}$, which is effectively an average of the relevant PN spin terms through the inspiral [78]. With only two spin parameters, they choose to write the final model in terms of the spin of only one of the black holes. That this is a reasonable choice (if not necessarily optimal; studies are ongoing) follows from the arguments above, and also from the results in [71], which show that the additional modulations in the dynamics in two-spin systems will be minor and in most cases not observable in gravitational-wave 
observations. The initial orientation of the spin in the plane is treated as an overall factor, which is the approximate degeneracy discussed in detail at the beginning of this section-however, this initial choice will in some cases be measurable in GW observations, allowing us to in principle track the direction of the orbital angular momentum through the inspiral [79].

PhenomP is a proof-of-principle model that involves many approximations: that the single-effective-spin approximation carries over to the precessional motion, that the precession effects can be parametrized by a single "precession spin" parameter $\chi_{p}$, that the stationary phase approximation (used to translate to the frequency domain) can be made through merger and ringdown, and that the $\mathrm{PN}$ expressions for the precession angles can be continued through merger and ringdown. The first two approximations are motivated by prior work, but the last two have no justification a priori. Nonetheless, when compared against a number of hybrid PN-NR waveforms, which included twospin configurations and configurations with a high degree of precession, the model performed well: it met the standard detection requirements (technically, a fitting factor with the signal higher than 0.97 ) for all but a small number of binary orientations.

The proposal of twisting non-precessing-binary waveforms has also been adopted to construct a generic EOB model [80]. Here the non-precessing-binary "SEOBNR" model is used as the underlying model. The precessional dynamics are produced by solving the EOB equations of motion for the precessing system. The reason for twisting up the underlying non-precessing-binary model, rather than calculating the waveform directly from the EOB dynamics using the results of Arun et al. [31], is that the more accurate EOB-factorized waveform modes are known only in the nonprecessing case. The resulting inspiral precession waveform is then connected to the ringdown waveform associated with the correct final spin, which is estimated, as in Ref. [77] by the empirical fits in Ref. [51]. The procedure to construct the inspiral precession waveform again relies on the observation of Ref. [75] that each precessing configuration has a specific non-precessing counterpart. As with the non-precessingbinary EOBNR model, this model does not make any reduction of the number of physical parameters: it uses all six spin components.

Beyond the approximations used to generate the inspiral waveforms, which are all well-motivated and backed up by studies of PN waveforms, the main open issue in these models is the treatment of the merger and ringdown. The results of Ref. [81] suggest that precession effects continue through the ringdown. The precession angles continue to evolve in PhenomP, but using only a naive continuation of the PN expressions used during the inspiral. As with typical frequency-domain PN expressions for the waveform phase, the expressions for the precession angles cannot be expected to be accurate through merger, but they do remain physically reasonable, and their accuracy does not have as strong an effect on the waveform as the phase. However, although these expressions appear to perform well in the comparisons in Ref. [77], it should be noted that in low-mass binaries most of the signal power is due to the inspiral, and so errors in the merger and ringdown have little effect on the overall agreement of two waveforms, while in high-mass binaries, where only the merger and ringdown will be detectable, there are very few GW cycles in the detectors' sensitivity band, and it is much easier to achieve a strong agreement after exploiting our freedom to make arbitrary relative time and phase shifts. 
In contrast to the PhenomP model, in the EOBNR model no precessional transformation is performed during the ringdown. We know from Ref. [81] that this is also incorrect, but, very likely for the same reasons as just argued above, the EOB model agrees well when compared against the two NR waveforms used for comparison in Ref. [80]. We should note, however, that these comparisons were performed with GW detection in mind, and the physical fidelity of these waveform models for parameter estimation remains to be studied in detail. It is very likely that such studies will highlight the aspects of these models that require improvement.

\section{Challenges and prospects}

There has been tremendous progress in the last few years in the modeling of gravitational waveforms from generic binaries. Until recently an accurate model of the full inspiral, merger and ringdown from generic binaries was considered a challenge that may not be met in time for the first GW detections. Now that has changed, and just in the last year two IMR models of generic binaries were proposed [77,80].

Nonetheless, a number of questions and challenges remain.

The current IMR models incorporate minimal physical information about the effects of precession on the merger and ringdown. It is not clear how difficult it will be to perform adequate numerical simulations to model those effects - can we again extract the main features from a subspace of the full binary parameter space, and if so, how does this subspace differ from that during the inspiral? In doing this, we must address the question of the required length of NR simulations. In non-precessing systems estimates of the required NR waveform lengths ranged from hundreds of orbits before merger if one wishes to eliminate all systematic bias from observations [82-84], down to tens of orbits if we allow small parameter biases at levels that are not expected to have any astrophysical impact $[85,86]$. It seems unlikely that long waveforms will be required to model the precession effects near merger, but no studies have yet addressed these questions. We must also ask how much of the merger physics will be detectable in GW observations. Although the merger is in the strong-field regime of Einstein's theory, the variations in the waveforms with respect to spin configurations are likely to be subtle and potentially invisible to ground-based detectors. It is important to clarify these issues, and prioritize the physical effects that need to be modelled, and efforts to do this are already underway $[65,81]$.

We should also note that the current generic IMR models consider only the $\ell=2$ harmonics of the waveforms. Although these are likely to be sufficient for detection, at least for comparable-mass binaries (for the non-precessing-binary case, see Ref. [87]), the effect on parameter estimation is yet to be fully understood.

In determining the necessary physical fidelity of the models, the impact on parameter estimation is likely to be the deciding factor. This also includes an understanding of the limits of parameter estimation inherent in the approximate degeneracies of the physical configurations. In non-precessing systems, we know that such a degeneracy between the mass ratio and spin will limit our ability to accurately measure the binary masses [88-91]. The efficacy of an effective-total-spin in the phenomenological [7,9] and PN [53] models tells us that it will be difficult to measure individual 
spins [64]. And our ability to parameterize precession effects with a single precession spin in Ref. [77] suggests that relative spin orientations will be difficult to determine. The details of these degeneracies need to be understood, not just to clarify the possibilities and limitations of astrophysical measurements from GW observations, but also to inform the regions of binary parameter space that most urgently need to be modeled.

All of these degeneracies are approximate, and can be disentangled in sufficiently strong signals. But we should bear in mind that the accuracy requirements of the theoretical models depends on the strength of the signals. By definition most observations with aLIGO and AdV will be close to the detection threshold of signal-to-noise ratio (SNR) 10. Less than $\sim 4 \%$ of observations will have an SNR above 30, and even at the most optimistic end of astrophysical event rate predictions, we will require several years of observation at design sensitivity before observing a signal with an SNR higher than 100 . On the other hand, when such an observation occurs, we should be ready to extract the maximum scientific information from it!

Associated with all of these issues is the question of how many NR waveforms are needed, and where in the parameter space they must come from. The answer to that question will likely emerge as part of the process of building waveform models: we won't know which waveforms we need until we can be sure that we don't need any more. Meanwhile, the production of NR waveforms proceeds at an encouraging pace. As striking examples, in the last year a collection of 224 generic waveforms were used in the study in Ref. [65], and a catalog of 174 generic waveforms was presented in Ref. [92]. In addition, the Numerical Injection Analysis (NINJA) collaboration [93] has produced a catalog of 64 PN-NR hybrids [94], which are being used to study the efficacy of search and parameter-estimation codes, and the Numerical-Relativity-Analytical-Relativity (NRAR) collaboration has performed an extensive study of the accuracy of a collection of 27 NR waveforms [95]. The NINJA study is currently limited to non-precessing binaries, and the quantification of waveform accuracies, and their requirements for modelling, remain problematic, but recent progress in numerical simulations is certainly comparable to the progress in modelling.

Beyond modelling issues, we also lack a search strategy for generic binaries, besides the first-approximation expectation that non-precessing-binary models will be sufficient to capture a large number of generic sources [96]. By definition a search that employed full generic-binary models would locate a wider range of signals, but it is possible that the increased false-alarm rate incurred by including more parameters in the search model would outweigh the better agreement between the generic-binary sources and the search templates.

In the long term (after 2025), if third-generation and space-based detectors come online $[97,98]$, then the situation may be quite different. As parameter degeneracies become less important and more accurate measurements become possible, the accuracy requirements of the waveform models will increase, and higher harmonics will be essential. It may also be possible at that stage to perform stringent tests of the general theory of relativity [99]; such tests will require far greater waveform precision than astrophysical measurements, although the details are yet to be clarified. 
Acknowledgments Thanks to Alejandro Bohé, Stephen Fairhurst, Maxime Fays, Sascha Husa, Frank Ohme, Francesco Pannarale, B. Sathyaprakash and Patricia Schmidt for useful discussions and/or comments on the manuscript. This work was supported by STFC grants ST/H008438/1 and ST/I001085/1.

Open Access This article is distributed under the terms of the Creative Commons Attribution License which permits any use, distribution, and reproduction in any medium, provided the original author(s) and the source are credited.

\section{References}

1. Harry, G.M.: Class. Quantum Gravity 27, 084006 (2010). doi:10.1088/0264-9381/27/8/084006

2. Acernese, F., et al.: Advanced virgo baseline design . Virgo technical document VIR-0027A-09 (2009)

3. Aasi, J., et al.: Prospects for localization of gravitational wave transients by the advanced LIGO and advanced virgo observatories (2013). arXiv:1304.0670

4. Abadie, J., et al.: Class. Quantum Gravity 27, 173001 (2010). doi:10.1088/0264-9381/27/17/173001

5. Blanchet, L.: Living Rev. Rel. 17, 2 (2014). doi:10.12942/lrr-2014-2

6. Centrella, J., Baker, J.G., Kelly, B.J., van Meter, J.R.: Rev. Mod. Phys. 82, 3069 (2010). doi:10.1103/ RevModPhys.82.3069

7. Ajith, P., Hannam, M., Husa, S., Chen, Y., Bruegmann, B., et al.: Phys. Rev. Lett. 106, 241101 (2011). doi:10.1103/PhysRevLett.106.241101

8. Pan, Y., Buonanno, A., Buchman, L.T., Chu, T., Kidder, L.E., et al.: Phys. Rev. D 81, 084041 (2010). doi:10.1103/PhysRevD.81.084041

9. Santamaria, L., Ohme, F., Ajith, P., Bruegmann, B., Dorband, N., et al.: Phys. Rev. D 82, 064016 (2010). doi:10.1103/PhysRevD.82.064016

10. Taracchini, A., Pan, Y., Buonanno, A., Barausse, E., Boyle, M., et al.: Phys. Rev. D 86, 024011 (2012). doi:10.1103/PhysRevD.86.024011

11. Taracchini, A., Buonanno, A., Pan, Y., Hinderer, T., Boyle, M., et al.: Phys. Rev. D89, 061502 (2014). doi:10.1103/PhysRevD.89.061502

12. Barker, B., O’Connell, R.: Phys. Rev. D 12, 329 (1975). doi:10.1103/PhysRevD.12.329

13. Barker, B., O’Connell, R.: Gen. Relativ. Gravit. 11, 149 (1979)

14. Damour, T.: The problem of motion in Newtonian and Einsteinian gravity. In: Hawking, S., Israel, W. (eds.) 300 Years of Gravitation. Cambridge University Press, Cambridge (1987)

15. Apostolatos, T.A., Cutler, C., Sussman, G.J., Thorne, K.S.: Phys. Rev. D 49, 6274 (1994). doi:10.1103/ PhysRevD.49.6274

16. Kidder, L.E.: Phys. Rev. D 52, 821 (1995). doi:10.1103/PhysRevD.52.821

17. Peters, P.: Phys. Rev. 136, B1224 (1964)

18. Buonanno, A., Chen, Yb, Vallisneri, M.: Phys. Rev. D 67, 104025 (2003). doi:10.1103/PhysRevD.67. 104025, doi:10.1103/PhysRevD.74.029904

19. Blanchet, L., Buonanno, A., Faye, G.: Phys. Rev. D 74, 104034 (2006). doi:10.1103/PhysRevD.81. 089901, doi:10.1103/PhysRevD.75.049903, doi:10.1103/PhysRevD.74.104034

20. Abadie, J., et al.: Phys. Rev. D 85, 082002 (2012). doi:10.1103/PhysRevD.85.082002

21. Aasi, J., et al.: Phys. Rev. D 87, 022002 (2013). doi:10.1103/PhysRevD.87.022002

22. Jaranowski, P., Schfer, G.: Phys. Rev. D 87, 081503 (2013). doi:10.1103/PhysRevD.87.081503

23. Hartung, J., Steinhoff, J.: Phys. Rev. D 83, 044008 (2011). doi:10.1103/PhysRevD.83.044008

24. Marsat, S., Bohé, A., Faye, G., Blanchet, L.: Class. Quantum Gravity 30, 055007 (2013). doi:10.1088/ 0264-9381/30/5/055007

25. Levi, M.: Phys. Rev. D 85, 064043 (2012). doi:10.1103/PhysRevD.85.064043

26. Hartung, J., Steinhoff, J.: Ann. Phys. 523, 919 (2011). doi:10.1002/andp.201100163

27. Bohé, A., Marsat, S., Blanchet, L.: Class. Quantum Gravity 30, 135009 (2013). doi:10.1088/ 0264-9381/30/13/135009

28. Marsat, S., Bohe, A., Blanchet, L., Buonanno, A.: Class. Quant. Grav. 31, 025023 (2014). doi:10.1088/ 0264-9381/31/2/025023

29. Gergely, L.A.: Phys. Rev. D 61, 024035 (2000). doi:10.1103/PhysRevD.61.024035

30. Faye, G., Marsat, S., Blanchet, L., Iyer, B.R.: (2012). arXiv:1210.2339

31. Arun, K., Buonanno, A., Faye, G., Ochsner, E.: Phys. Rev. D 79, 104023 (2009). doi:10.1103/ PhysRevD.79.104023, doi:10.1103/PhysRevD.84.049901 
32. Buonanno, A., Iyer, B., Ochsner, E., Pan, Y., Sathyaprakash, B.: Phys. Rev. D 80, 084043 (2009). doi:10.1103/PhysRevD.80.084043

33. Nitz, A.H., Lundgren, A., Brown, D.A., Ochsner, E., Keppel, D., et al.: Phys. Rev. D88, 124039 (2013). doi:10.1103/PhysRevD.88.124039

34. Brown, D.A., Harry, I., Lundgren, A., Nitz, A.H.: Phys. Rev. D 86, 084017 (2012). doi:10.1103/ PhysRevD.86.084017

35. Hannam, M., Husa, S., Sperhake, U., Bruegmann, B., Gonzalez, J.A.: Phys. Rev. D 77, 044020 (2008). doi:10.1103/PhysRevD.77.044020

36. Boyle, M., Brown, D.A., Kidder, L.E., Mroue, A.H., Pfeiffer, H.P., et al.: Phys. Rev. D 76, 124038 (2007). doi:10.1103/PhysRevD.76.124038

37. Hannam, M., Husa, S., Bruegmann, B., Gopakumar, A.: Phys. Rev. D 78, 104007 (2008). doi:10.1103/ PhysRevD.78.104007

38. Hannam, M., Husa, S., Ohme, F., Muller, D., Bruegmann, B.: Phys. Rev. D 82, 124008 (2010). doi:10. 1103/PhysRevD.82.124008

39. MacDonald, I., Mroue, A.H., Pfeiffer, H.P., Boyle, M., Kidder, L.E., et al.: Phys. Rev. D 87, 024009 (2013). doi:10.1103/PhysRevD.87.024009

40. Buonanno, A., Damour, T.: Phys. Rev. D 59, 084006 (1999). doi:10.1103/PhysRevD.59.084006

41. Buonanno, A., Damour, T.: Phys. Rev. D 62, 064015 (2000). doi:10.1103/PhysRevD.62.064015

42. Damour, T.: Phys. Rev. D 64, 124013 (2001). doi:10.1103/PhysRevD.64.124013

43. Buonanno, A., Chen, Y., Damour, T.: Phys. Rev. D 74, 104005 (2006). doi:10.1103/PhysRevD.74. 104005

44. Pretorius, F.: Phys. Rev. Lett. 95, 121101 (2005). doi:10.1103/PhysRevLett.95.121101

45. Campanelli, M., Lousto, C.O., Marronetti, P., Zlochower, Y.: Phys. Rev. Lett. 96, 111101 (2006). doi:10.1103/PhysRevLett.96.111101

46. Baker, J.G., Centrella, J., Choi, D.I., Koppitz, M., van Meter, J.: Phys. Rev. Lett. 96, 111102 (2006). doi:10.1103/PhysRevLett.96.111102

47. Ajith, P., Babak, S., Chen, Y., Hewitson, M., Krishnan, B., et al.: Class. Quantum Gravity 24, S689 (2007). doi:10.1088/0264-9381/24/19/S31

48. Buonanno, A., Cook, G.B., Pretorius, F.: Phys. Rev. D 75, 124018 (2007). doi:10.1103/PhysRevD.75. 124018

49. Buonanno, A., Kidder, L.E., Lehner, L.: Phys. Rev. D 77, 026004 (2008). doi:10.1103/PhysRevD.77. 026004

50. Hemberger, D.A., Lovelace, G., Loredo, T.J., Kidder, L.E., Scheel, M.A., et al.: Phys. Rev. D 88, 064014 (2013). doi:10.1103/PhysRevD.88.064014

51. Barausse, E., Rezzolla, L.: Astrophys. J. 704, L40 (2009). doi:10.1088/0004-637X/704/1/L40

52. Poisson, E., Will, C.M.: Phys. Rev. D 52, 848 (1995). doi:10.1103/PhysRevD.52.848

53. Ajith, P.: Phys. Rev. D 84, 084037 (2011). doi:10.1103/PhysRevD.84.084037

54. Ajith, P., Babak, S., Chen, Y., Hewitson, M., Krishnan, B., et al.: Phys. Rev. D 77, 104017 (2008). doi:10.1103/PhysRevD.79.129901, doi:10.1103/PhysRevD.77.104017

55. Buonanno, A., Pan, Y., Baker, J.G., Centrella, J., Kelly, B.J., et al.: Phys. Rev. D 76, 104049 (2007). doi:10.1103/PhysRevD.76.104049

56. Damour, T., Iyer, B.R., Nagar, A.: Phys. Rev. D 79, 064004 (2009). doi:10.1103/PhysRevD.79.064004

57. Pan, Y., Buonanno, A., Fujita, R., Racine, E., Tagoshi, H.: Phys. Rev. D 83, 064003 (2011). doi:10. 1103/PhysRevD.83.064003

58. Damour, T., Nagar, A., Bernuzzi, S.: Phys. Rev. D 87, 084035 (2013). doi:10.1103/PhysRevD.87. 084035

59. Pan, Y., Buonanno, A., Boyle, M., Buchman, L.T., Kidder, L.E., et al.: Phys. Rev. D 84, 124052 (2011). doi:10.1103/PhysRevD.84.124052

60. Barausse, E., Buonanno, A.: Phys. Rev. D 81, 084024 (2010). doi:10.1103/PhysRevD.81.084024

61. Field, S.E., Galley, C.R., Hesthaven, J.S., Kaye, J., Tiglio, M.: Phys. Rev. X4, 031006 (2014). doi:10. 1103/PhysRevX.4.031006

62. Pürrer, M.: (2014). arXiv:1402.4146

63. Cannon, K., Emberson, J., Hanna, C., Keppel, D., Pfeiffer, H.: Phys. Rev. D 87(4), 044008 (2013). doi:10.1103/PhysRevD.87.044008

64. Pürrer, M., Hannam, M., Ajith, P., Husa, S.: Phys. Rev. D 88, 064007 (2013). doi:10.1103/PhysRevD. 88.064007 
65. Pekowsky, L., O’Shaughnessy, R., Healy, J., Shoemaker, D.: Phys. Rev. D 88, 024040 (2013). doi:10. 1103/PhysRevD.88.024040

66. Gonzalez, J., Hannam, M., Sperhake, U., Bruegmann, B., Husa, S.: Phys. Rev. Lett. 98, 231101 (2007). doi:10.1103/PhysRevLett.98.231101

67. Campanelli, M., Lousto, C.O., Zlochower, Y., Merritt, D.: Phys. Rev. Lett. 98, 231102 (2007). doi:10. 1103/PhysRevLett.98.231102

68. Bruegmann, B., Gonzalez, J.A., Hannam, M., Husa, S., Sperhake, U.: Phys. Rev. D 77, 124047 (2008). doi:10.1103/PhysRevD.77.124047

69. Sturani, R., Fischetti, S., Cadonati, L., Guidi, G., Healy, J., et al.: J. Phys. Conf. Ser. 243, 012007 (2010). doi:10.1088/1742-6596/243/1/012007

70. Apostolatos, T.: Phys. Rev. D 52, 605 (1995). doi:10.1103/PhysRevD.52.605

71. Pan, Y., Buonanno, A., Chen, Yb, Vallisneri, M.: Phys. Rev. D 69, 104017 (2004). doi:10.1103/ PhysRevD.69.104017, doi:10.1103/PhysRevD.74.029905

72. Schmidt, P., Hannam, M., Husa, S., Ajith, P.: Phys. Rev. D 84, 024046 (2011). doi:10.1103/PhysRevD. 84.024046

73. O’Shaughnessy, R., Vaishnav, B., Healy, J., Meeks, Z., Shoemaker, D.: Phys. Rev. D 84, 124002 (2011). doi:10.1103/PhysRevD.84.124002

74. Boyle, M., Owen, R., Pfeiffer, H.P.: Phys. Rev. D 84, 124011 (2011). doi:10.1103/PhysRevD.84. 124011

75. Schmidt, P., Hannam, M., Husa, S.: Phys. Rev. D 86, 104063 (2012). doi:10.1103/PhysRevD.86. 104063

76. Lundgren, A., O’Shaughnessy, R.: Phys. Rev. D89, 044021 (2014). doi:10.1103/PhysRevD.89.044021

77. Hannam, M., Schmidt, P., Bohé, A., Haegel, L., Husa, S., et al.: (2013). arXiv:1308.3271

78. Schmidt, P., Hannam, M., Ohme, F.: In preparation. (2013). arXiv:1408.1810

79. O’Shaughnessy, R., Farr, B., Ochsner, E., Cho, H., Raymond, V., et al.: (2014). arXiv:1403.0544

80. Pan, Y., Buonanno, A., Taracchini, A., Kidder, L.E., Mroue, A.H., et al.: Phys. Rev. D89, 084006 (2014). doi:10.1103/PhysRevD.89.084006

81. O’Shaughnessy, R., London, L., Healy, J., Shoemaker, D.: Phys. Rev. D 87(4), 044038 (2013). doi:10. 1103/PhysRevD.87.044038

82. MacDonald, I., Nissanke, S., Pfeiffer, H.P.: Class. Quant. Gravity 28, 134002 (2011). doi:10.1088/ 0264-9381/28/13/134002

83. Damour, T., Nagar, A., Trias, M.: Phys. Rev. D 83, 024006 (2011). doi:10.1103/PhysRevD.83.024006

84. Boyle, M.: Phys. Rev. D 84, 064013 (2011). doi:10.1103/PhysRevD.84.064013

85. Hannam, M., Husa, S., Ohme, F., Ajith, P.: Phys. Rev. D 82, 124052 (2010). doi:10.1103/PhysRevD. 82.124052

86. Ohme, F., Hannam, M., Husa, S.: Phys. Rev. D 84, 064029 (2011). doi:10.1103/PhysRevD.84.064029

87. Capano, C., Pan, Y., Buonanno, A.: Phys. Rev. D89, 102003 (2014). doi:10.1103/PhysRevD.89.102003

88. Cutler, C., Flanagan, E.E.: Phys. Rev. D 49, 2658 (1994). doi:10.1103/PhysRevD.49.2658

89. Baird, E., Fairhurst, S., Hannam, M., Murphy, P.: Phys. Rev. D 87, 024035 (2013). doi:10.1103/ PhysRevD.87.024035

90. Hannam, M., Brown, D.A., Fairhurst, S., Fryer, C.L., Harry, I.W.: Astrophys. J. 766, L14 (2013). doi:10.1088/2041-8205/766/1/L14

91. Ohme, F., Nielsen, A.B., Keppel, D., Lundgren, A.: Phys. Rev. D 88, 042002 (2013). doi:10.1103/ PhysRevD.88.042002

92. Mroue, A.H., Scheel, M.A., Szilagyi, B., Pfeiffer, H.P., Boyle, M., et al.: Phys. Rev. Lett. 111, 241104 (2013). doi:10.1103/PhysRevLett.111.241104

93. Aylott, B., Baker, J.G., Boggs, W.D., Boyle, M., Brady, P.R., et al.: Class. Quantum Gravity 26, 165008 (2009). doi:10.1088/0264-9381/26/16/165008

94. Ajith, P., Boyle, M., Brown, D.A., Brgmann, B., Buchman, L.T., et al.: Class. Quantum Gravity 29, 124001 (2012). doi:10.1088/0264-9381/30/19/199401, doi:10.1088/0264-9381/29/12/124001

95. Hinder, I., Buonanno, A., Boyle, M., Etienne, Z.B., Healy, J., et al.: Class. Quantum Gravity 31, 025012 (2013). doi:10.1088/0264-9381/31/2/025012

96. Harry, I., Nitz, A., Brown, D.A., Lundgren, A., Ochsner, E., et al.: Phys. Rev. D89, 024010 (2014). doi:10.1103/PhysRevD.89.024010

97. Punturo, M., Abernathy, M., Acernese, F., Allen, B., Andersson, N., et al.: Class. Quantum Gravity 27, 194002 (2010). doi:10.1088/0264-9381/27/19/194002

98. Seoane, P.A., et al.: (2013). arXiv:1305.5720 
99. Yunes, N., Siemens, X.: Living Rev. Relat. 16(9). DOI:10.12942/lrr-2013-9. http://www.livingreviews. org/lrr-2013-9 (2013) 